\title{
Giant electrostrain accompanying structural evolution in lead-free NBT-based piezoceramics
}

\begin{tabular}{|r|l|}
\hline Journal: & Journal of Materials Chemistry C \\
\hline Manuscript ID & TC-ART-11-2017-005359.R1 \\
\hline Article Type: & Paper \\
\hline Date Submitted by the Author: & 08-Dec-2017 \\
\hline Complete List of Authors: & $\begin{array}{l}\text { Liu, Xing; Tongji University, School of Materials Science \& Engineering } \\
\text { Xue, Saidong; Shanghai Normal University } \\
\text { Li, Feng; Tongji University College of Sciences, Materials Science } \\
\text { Ma, Jinpeng; Shanghai Normal University } \\
\text { Zhai, Jiwei; Tongji University, School of Materials Science \& Engineering } \\
\text { Shen, Bo; Tongji University, School of Materials Science \& Engineering } \\
\text { Wang, Feifei; Shanghai Normal University, Physics } \\
\text { Zhao, Xiangyong; Shanghai Normal University, Key Laboratory of } \\
\text { Optoelectronic Material and Device, Department of Physics } \\
\text { Yan, Haixue; Queen Mary University of London, School of Engineering and } \\
\text { Materials Science }\end{array}$ \\
\hline \multicolumn{2}{|l}{} \\
\hline
\end{tabular}




\section{Journal Name}

\section{Giant electrostrain accompanying structural evolution in lead-free NBT-based piezoceramics}

Received 00th January 20xx, Accepted 00th January 20xx

DOI: $10.1039 / \times 0 \times x 00000 x$

www.rsc.org/

\author{
Xing Liu ${ }^{a}$, Saidong Xue ${ }^{b}$, Feng $\mathrm{Li}^{\mathrm{a}}$, Jinpeng Ma ${ }^{\mathrm{b}}$, Jiwei Zhai ${ }^{\mathrm{a}, *}$, Bo Shen ${ }^{\mathrm{a}}$, Feifei Wang ${ }^{\mathrm{b}}$, Xiangyong \\ Zhao ${ }^{b}$, Haixue Yan ${ }^{c, *}$
}

High-performance $\left(\mathrm{Na}_{0.5} \mathrm{Bi}_{0.5}\right) \mathrm{TiO}_{3}$ (NBT) lead-free incipient ceramics are promising piezoactuator materials, but high driving fields to deliver the large strain limits their practical applications. Herein, we report a giant piezoelectric strain $\left(d_{33}{ }^{*}\right)$ of $810 \mathrm{pm} / \mathrm{V}$ at a low driving field of $4 \mathrm{kV} / \mathrm{mm}$ in a novel ternary $(0.94-\mathrm{x})\left(\mathrm{Na}_{0.5} \mathrm{Bi}_{0.5}\right) \mathrm{TiO}_{3-}$ $0.06 \mathrm{Ba}\left(\mathrm{Zr}_{0.05} \mathrm{Ti}_{0.95}\right) \mathrm{O}_{3}-\mathrm{x}\left(\mathrm{Sr}_{0.8} \mathrm{Bi}_{0.1} \square_{0.1}\right) \mathrm{TiO}_{2.95}$ solid solution with $\mathrm{x}=0.05$ (SBT5). The field of SBT5 critical composition is notably reduced compared with other NBT-based ceramics, while its strain property maintains at a high level along with an excellent thermal stability. The dopant induces a randomly distributed local polarization field, which breaks the symmetry of Landau potential curves, boosts the ferroelectric instability and favors a more disordered relaxor structure. The giant strain in the critical composition SBT5 is due to a field-induced reversible relaxor-ferroelectric phase transformation, while the reduced driving field results from two synergistic effects: remanent quasiferroelectric order as the seed for the growth of polar domains helps the system skip nucleation process; the local defects further facilitate the growth of ferroelectric domains. The composition, temperature and electric-field dependence of structural evolutions were systematically elucidated from micro- and macroscopic view. This study opens up a feasible and effective way for achieving a giant electrostrain in lead-free actuator materials.

\section{Introduction}

Piezoelectric materials can achieve direct conversion between mechanical and electrical energy. ${ }^{1}$ Lead-based $\mathrm{Pb}(\mathrm{Zr}, \mathrm{Ti}) \mathrm{O}_{3}$ (PZT) piezoceramics with the morphotropic phase boundary (MPB) composition exhibit salient functional properties, and the versatility of PZT makes it irreplaceable in global piezoelectric market. ${ }^{1,2}$ However, due to the toxicity of lead and growing concerns on environmental protection and human health, tremendous efforts have been focused on developing sustainable lead-free piezoceramics that possess comparable electromechanical properties to those of leadcontaining materials. ${ }^{3-5}$

Recent intense research on lead-free piezoceramics has led to the discovery of many ferroelectric oxides with excellent physical properties. ${ }^{3-5}$ Among all the lead-free systems developed, $\left(\mathrm{Na}_{0.5} \mathrm{Bi}_{0.5}\right) \mathrm{TiO}_{3}$ (NBT) has drawn considerable

\footnotetext{
Key laboratory of Advanced Civil Engineering Materials of Ministry of Education, Functional Materials Research Laboratory, School of Materials Science \& Engineering, Tongji University, 4800 Caoan Road, Shanghai 201804, China

${ }^{b}$. Key Laboratory of Optoelectronic Material and Device, Department of Physics, Shanghai Normal University, Shanghai, 200234, China.

School of Engineering and Materials Science, Queen Mary University of London, Mile End Road, London E1 4NS, UK.

E-mail addresses of corresponding authors: apzhai@tongji.edu.cn (J. Zhai); h.x.yan@qmul.ac.uk (H. Yan).

Electronic Supplementary Information (ESI) available. See
} DOI: $10.1039 / \times 0 \times x 00000 x$ attention, since the $\mathrm{Bi}^{3+}$ ion owns large polarizability caused by the $6 \mathrm{~s}^{2}$ lone-pair electrons that could contribute to a large structural distortion and high ferroelectricity. ${ }^{5-7}$ NBT can form solid solutions with other $\mathrm{ABO}_{3}$-type ferroelectrics, such as NBT-BaTiO, NBT-Ba(Zr, Ti)O, NBT-(K, Na)NbO ${ }_{3}, \mathrm{NBT}_{3} \mathrm{SrTiO}_{3}$, etc. $^{8-11}$ Indeed, the origin of high piezoelectric response in these MPB compositions lies in the existence of monoclinic nanotwins (nanodomains) as an intermediate bridging phase, which facilitates a low-energy pathway for polarization rotation. ${ }^{12}$ Furthermore, an exceptionally large recoverable strain of $\sim 0.4 \%$ has been achieved in NBT-based incipient ceramics, making them promising candidates for applications in actuators. ${ }^{5,13,14}$ Via chemical modifications, the long-range polar order of NBT-based systems can be disrupted and the ergodic relaxor phase will present in the vicinity of room temperature. ${ }^{5}$ Recent studies have highlighted that the origin of the large strain is due to a reversible field-induced relaxorto-ferroelectric phase transformation. ${ }^{15,16}$ However, the electric fields needed to trigger the large strain properties are as high as $6 \sim 9 \mathrm{kV} / \mathrm{mm}$, resulting in a low $d_{33}{ }^{*}$ (large-signal piezoelectric coefficient). ${ }^{5,13,14}$ Therefore, how to reduce the driving field while maintain a high strain performance and high stability remains a big challenge for practical actuator applications.

In this work, $0.94\left(\mathrm{Na}_{0.5} \mathrm{Bi}_{0.5}\right) \mathrm{TiO}_{3}-0.06 \mathrm{Ba}\left(\mathrm{Zr}_{0.05} \mathrm{Ti}_{0.95}\right) \mathrm{O}_{3}$ (0.94NBT-0.06BZT) was chosen as the base composition, $\left(\mathrm{Sr}_{0.8} \mathrm{Bi}_{0.1} \square_{0.1}\right) \mathrm{TiO}_{2.95}$ (SBT, $\square$ represents the A-site vacancy) as 
the modifier, and a novel ternary (0.94-x)NBT-0.06BZT-xSBT system was constructed. $\left(\mathrm{Sr}_{0.8} \mathrm{Bi}_{0.1} \square_{0.1}\right) \mathrm{TiO}_{2.95}$ is a typical A-site heterovalent-doped relaxor ferroelectrics with the A-site vacancies $\left(V_{\mathrm{A}}\right)$ and oxygen vacancies $\left(V_{\mathrm{O}}\right)$ produced to balance the charge misfit between $\mathrm{Sr}^{2+}$ and $\mathrm{Bi}^{3+}$ ions. ${ }^{17}$ The local defects $\left(V_{\mathrm{A}}\right.$ and $\left.V_{\mathrm{O}}\right)$ were simultaneously introduced into the system to create a randomly distributed local polarization field, which breaks the symmetry of Landau potential curves and disrupts the long-range polar order. ${ }^{18}$ More intriguingly, we achieved a giant $d_{33}{ }^{*}$ value of $810 \mathrm{pm} / \mathrm{V}$ at a low driving field of $4 \mathrm{kV} / \mathrm{mm}$ in SBT5 composition. Furthermore, systematic electrical and structural characterizations were carried out to reveal the physical origin of the high performances.

\section{Experimental}

\subsection{Sample preparation}

(0.94-x) $\left(\mathrm{Na}_{0.5} \mathrm{Bi}_{0.5}\right) \mathrm{TiO}_{3}-0.06 \mathrm{Ba}\left(\mathrm{Zr}_{0.05} \mathrm{Ti}_{0.95}\right) \mathrm{O}_{3}-$ $\mathrm{x}\left(\mathrm{Sr}_{0.8} \mathrm{Bi}_{0.1} \square_{0.1}\right) \mathrm{TiO}_{2.95}$ with $\mathrm{x}=0-10 \mathrm{~mol} \%$ (abbreviated as SBTOSBT10) were prepared by a conventional solid-state reaction method. The starting materials, including $\mathrm{Na}_{2} \mathrm{CO}_{3}, \mathrm{Bi}_{2} \mathrm{O}_{3}, \mathrm{SrCO}_{3}$, $\mathrm{BaCO}_{3}, \mathrm{ZrO}_{2}$ and $\mathrm{TiO}_{2}$ (purity>99.9\%) were weighed and ball milled for $12 \mathrm{~h}$. The dried slurries were calcined at $850^{\circ} \mathrm{C}$ for 5 $\mathrm{h}$ and then remilled again for $12 \mathrm{~h}$ to enhance the compositional homogenization. The dried powders were pressed into pellets of $10 \mathrm{~mm}$ in diameter using 8 wt\% polyvinyl alcohol as the binder. The pellets were heated to 550 ${ }^{\circ} \mathrm{C}$ for $10 \mathrm{~h}$ to burn off the binder. Then the green pellets were embedded in precursor powders and sintered at $1140 \sim 1160^{\circ} \mathrm{C}$ for $3 \mathrm{~h}$ in a covered alumina crucible. The ceramic samples were polished to a thickness of $0.4 \mathrm{~mm}$. Prior to structure and property measurements, all the samples were annealed at 420 ${ }^{\circ} \mathrm{C}$ for $2 \mathrm{~h}$ to release any pre-stress.

\subsection{Structural measurements}

The density was measured using the Archimedes method. The powder X-ray diffraction measurements (XRD, Bruker D8 Advanced, Germany) with $\mathrm{Cu} K \alpha$ radiation were carried out to detect the crystal structure. Surface morphologies were observed by the SEM (JSM, EMP-800, JEOL, Tokoyo, Japan). Raman spectroscopy was recorded using a Horiba Lab-Ram HR800 spectrometer equipped with a Linkam THM 600 heating stage. The domain relaxation was characterized by piezoresponse force microscopy (PFM, MFP-3D, Asylum Research, USA). Before PFM observations, the samples were carefully polished using polycrystalline diamond paste with particle size of $15 \mu \mathrm{m}, 6 \mu \mathrm{m}, 3 \mu \mathrm{m}, 1 \mu \mathrm{m}$ and $0.25 \mu \mathrm{m}$ for $1 \mathrm{~h}$ each. The root mean square (RMS) of all samples was less than $1 \mathrm{~nm}$.

\subsection{Electrical measurements}

For electrical characterizations, the silver paste was coated on both surfaces of ceramic samples and fired at $520{ }^{\circ} \mathrm{C}$ for 30 $\min$. The specimens were poled under a dc field of $5 \mathrm{kV} / \mathrm{mm}$ for $15 \mathrm{~min}$. Dielectric properties and impedance spectra were measured using a computer-controlled LCR meter (Agilent E4980A, Agilent, Palo Alto, CA). Thermally stimulated depolarization current measurement was carried out using a Keithley 6517A ampere meter (Keithley Instruments, Cleveland, $\mathrm{OH}$ ). The polarization and strain curves were tested by a FE analyzer connected with a laser interferometer (TF2000, aix-ACCT, Aachen, Germany).

\section{Results and discussion}

\subsection{Phase identification and microstructure}

The SEM morphologies (Fig. S1) reveal that the synthesized ceramics have a highly compact and uniform microstructure with the relative densities of $\sim 97 \%$ and average grain size of $\sim 0.75 \mu \mathrm{m}$. A pure perovskite structure with no trace of secondary phase was detected for all compositions as shown in Fig. S2(a), suggesting that the SBT has been completely diffused into NBT-BZT host lattice and forms solid solutions. Sharp and narrow diffraction peaks reveal a pseudocubic symmetry for all compositions, and the close inspection of (111) and (200) characteristic peaks (Fig. S2(b)) further indicates that there is no obvious non-cubic distortion. Due to the relatively large ionic radii of $\mathrm{Sr}^{2+}$ compared with $\mathrm{Bi}^{3+}$ and

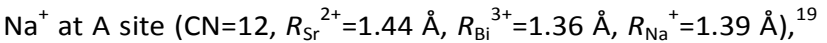
the substitution induces a slight lattice expansion (Fig. S2(c)) and the diffraction peaks shift to lower angles. Despite the apparent absence of long-range distortion, it is reasonable to assume that the symmetry should be distorted from cubic on a local scale, ${ }^{6,7}$ as will be subsequently discussed.

\subsection{Electromechanical properties}

Fig. 1(a)-(c) show the bipolar $P-E$ hysteresis loops, bipolar and unipolar strain curves of SBT0-SBT10 samples measured at 6 $\mathrm{kV} / \mathrm{mm}$ and $10 \mathrm{~Hz}$. The polarization $\left(P_{\max }, P_{\mathrm{r}}\right.$ and $\left.P_{\max }-P_{\mathrm{r}}\right)$ and strain values $\left(S_{\text {pos }}, S_{\text {neg }}, d_{33}{ }^{*}\right.$ and $\left.d_{33}\right)$ were summarized in Fig. S3. With the increase of SBT content, the $P-E$ loops change from well-saturated shape to pinched shape and the bipolar strain curves change from butterfly shape to sprout shape, coupled with the gradual reduction of $P_{\max }$ and $P_{\mathrm{r}}$ values and the increase of $P_{\max }-P_{\mathrm{r}}$ values. Such phenomenon demonstrates that the SBT substitution disrupts the long-range ferroelectric state and induces a ferroelectric-to-relaxor transition. ${ }^{15}$ The $S_{\text {neg }}$ and $d_{33}$ values, which are correlated with the domain back-switching, ${ }^{5,15}$ drastically deteriorate at SBT5 composition. Meanwhile, the $S_{\text {pos }}$ and $d_{33}{ }^{*}$ values first increase, reach the maximum of $0.51 \%$ and $750 \mathrm{pm} / \mathrm{V}$ at SBT5 composition, and then decrease as SBT content further increases. Similar phenomenon has also been reported in other NBT-based incipient ceramics, of which the emergence 
of giant recoverable strains is always accompanied by the disturbance of macro-ferroelectricity. ${ }^{20-24}$ To evaluate the suitability of SBT5 sample for practical actuator applications, the unipolar strain curves under different electric fields were measured and are presented in Fig. 1 (d). The $d_{33}{ }^{*}$ value of SBT5 sample increases from $190 \mathrm{pm} / \mathrm{V}$ at $2 \mathrm{kV} / \mathrm{mm}$ to $810 \mathrm{pm} / \mathrm{V}$ at 4 $\mathrm{kV} / \mathrm{mm}$, then decreases as the field further increases. The slight decrease of $d_{33}{ }^{*}$ value at high fields is due to the fact that the $d_{33}{ }^{*}$ is calculated by the average unipolar strain per unit of field: $d_{33}{ }^{*}=S_{\text {unipolar }} / E$, and the unipolar strain of SBT5 sample has already reached saturation at a low field. ${ }^{24} \mathrm{Fig} .1(\mathrm{e})$ shows the $d_{33}{ }^{*}$ values vs. driving fields of some widely studied actuator materials, including PZT, Bi-based $\mathrm{Bi}\left(\mathrm{Mg}_{0.5} \mathrm{Ti}_{0.5}\right) \mathrm{O}_{3}$ (BMT)- and $\mathrm{Bi}\left(\mathrm{Ni}_{0.5} \mathrm{Ti}_{0.5}\right) \mathrm{O}_{3}$ (BNiT)-, and $\mathrm{K}_{0.5} \mathrm{Na}_{0.5} \mathrm{NbO}_{3}(\mathrm{KNN})$ based systems. ${ }^{14,25-31}$ The strain properties of other NBT-based incipient materials are also summarized in Fig. S4. Note that only the piezoceramics prepared by traditional solid-state sintering method are listed for comparison. Although the single crystals or grain-oriented ceramics possess remarkable electrostrains, the high cost and difficulty in fabrication would limit their production in quantity. The SBT5 composition possesses a large $d_{33}{ }^{*}$ value of $810 \mathrm{pm} / \mathrm{V}$ at a low driving field of $4 \mathrm{kV} / \mathrm{mm}$, which competes with commercial PZT ceramics and surpasses other Bi-based and KNN-based ceramics. ${ }^{14,25-31}$ Moreover, the $d_{33}{ }^{*}$ value of SBT5 sample presents an excellent thermal stability with the variation less than $11 \%$ up to $80{ }^{\circ} \mathrm{C}$ (Fig. S5(I)), showing a great potential for piezoactuator applications.

To further investigate the electric field dependence of polarization reorientation and strain behavior, the $\mathrm{d} S / \mathrm{d} E$ values of the lower-half unipolar strain curves during electric loading were calculated, as shown in Fig. 1(f). The $d S / d E$ curves of SBTO-SBT4 samples display no observable changes, while a sharp peak appears at $\sim 3 \mathrm{kV} / \mathrm{mm}$ for SBT5 sample, and this peak shifts to higher field region and becomes more diffuse with further increase of SBT content. The threshold field of the peak on $d S / d E$ curve is defined as the activating field at which the fast increase of strain in response to the field occurs, and the intensity of this peak is closely related to the velocity of the oriented growth of polar-nano-regions (PNRs). ${ }^{32}$ The unipolar strain of SBT5 sample presents a significant enhancement at $\sim 3 \mathrm{kV} / \mathrm{mm}$ and saturates at $\sim 4 \mathrm{kV} / \mathrm{mm}$, deriving a low-field driven high electrostrain. Moreover, the higher $\mathrm{d} S / \mathrm{d} E$ peak intensity and lower activating field of SBT5 composition should be attributed to the lower energy barrier for polarization switching near the ferroelectric-relaxor phase boundary, ${ }^{32}$ which will be discussed later in detail.

The temperature dependent ferroelectric responses of three representative compositions (SBTO, SBT2 and SBT5) were also measured to study the temperature induced structural evolutions, and are shown in Fig. S5. The composition- and temperature-dependence of polarization and strain curves demonstrate a similar behavior, i.e., the destabilization of long-range ferroelectricity and a pronounced enhancement of strain response at critical composition or temperature, suggesting the occurrence of ferroelectric-relaxor transition. ${ }^{15,16}$

\subsection{Dielectric analyses}

To identify the phase structural evolutions, the temperature dependence of dielectric properties of all poled samples during the heating and cooling processes were measured and are displayed in Fig. S6. The cooling data can be considered as that of unpoled one since the sample loses the poling-related memory after heating to a sufficiently high temperature. Fig. 2(a) shows the dielectric spectra of poled SBTO-SBT5 samples during heating process. Two diffused dielectric peaks locate at $\sim 120{ }^{\circ} \mathrm{C}\left(T_{\mathrm{s}}\right)$ and $\sim 270{ }^{\circ} \mathrm{C}\left(T_{\mathrm{m}}\right)$ can be observed for all compositions. Previous studies revealed that the two broad dielectric peaks are associated with the thermal evolutions of rhombohedral and tetragonal PNRs. ${ }^{33}$ Furthermore, a discernible difference between the heating and cooling dielectric spectra is revealed by a lower-temperature sharp anomaly. This anomaly actually corresponds to the thermotropic phase boundary between ferroelectric and relaxor phase (marked by $\left.T_{\mathrm{FR}}\right)^{33}$ The $T_{\mathrm{FR}}$ obtained from dielectric measurement has also been referred to as depolarization temperature $\left(T_{\mathrm{d}}\right) \cdot{ }^{34}$ Fig. $2(\mathrm{~b})$ displays the temperature dependence of depolarization current $\left(j_{\text {TSDC }}\right)$ for poled SBTO-SBT5 samples, and $T_{\mathrm{d}}$ is determined from the peak in $j_{\text {TSDC. }}$ Fig. 2(c) summarizes the $T_{m}, T_{\mathrm{FR}}$ and $T_{\mathrm{d}}$ of all compositions. It can be seen that $T_{\mathrm{d}}$ is slightly lower than $T_{\mathrm{FR}}$. This discrepancy is due to the fact that the ferroelectric-relaxor transition consists of two separate processes: the domain randomization at $T_{\mathrm{d}}$, followed by the break-up of ferroelectric domains into discrete PNRs at $T_{\mathrm{FR}} \cdot{ }^{34}$ Generally, both the $T_{\mathrm{FR}}$ and $T_{\mathrm{d}}$ decrease progressively with increasing SBT content and disappear at SBT5 composition, while $T_{\mathrm{m}}$ displays no obvious changes. The downward shift of $T_{\mathrm{FR}}$ and $T_{\mathrm{d}}$ with SBT substitution suggests a composition-induced ferroelectricrelaxor transition and SBT5 is considered as critical composition, $5,15,33$ which is in good agreement with the polarization and strain measurements aforementioned.

The dielectric constant above $T_{m}$ was fitted by the CurieWeiss law and quadratic law to further investigate the dielectric relaxor behavior:

$\varepsilon=C /\left(T-T_{\mathrm{m}}\right)$, Curie-Weiss law

$1 / \varepsilon=1 / \varepsilon_{\mathrm{m}}+\left(T-T_{\mathrm{m}}\right)^{n} / C^{\prime}$, quadratic law

where $C$ and $C^{\prime}$ are constant; $n$ is a quantitative measure of diffuseness, $n$ is 1 for normal ferroelectrics, whereas $n$ is 2 for ideal relaxor ferroelectrics. ${ }^{35}$ The fitted dielectric spectra of SBT0, SBT5 and SBT10 compositions are shown in Fig. S7. It is clearly that the dielectric constant more obviously deviates from the Curie-Weiss law with increasing SBT content, and this deviation is defined by the $\Delta T$ value: $\Delta T=T_{\mathrm{B}}-T_{\mathrm{m}}$, where $T_{\mathrm{B}}$ is Burns temperature from which the dielectric constant starts to deviate from the Curie-Weiss law. ${ }^{35}$ Therefore the relaxor degree can be roughly evaluated by $n$ and $\Delta T$ values. As shown 
in Fig. 2(d), the $n$ and $\Delta T$ values increase continuously with SBT substitution, suggesting that SBT enhances the relaxor degree and favors a more disordered structure. ${ }^{35}$

\subsection{Local phenomena}

As opposed to X-ray diffraction that provides data on average crystal structure, Raman spectroscopy has been proven to be a sensitive tool in resolving the local structure on a short range owing to its coherence length down to the nanometer scale. $6,7,36$ The piezoresponse force microscopy (PFM) is another powerful tool in determining the local phenomena of ferroelectrics. PFM could provide an insight into the domain structure and local polarization switching when an external field is applied to a conductive tip.. ${ }^{37,38}$ The gathered information from PFM combined with Raman spectra is crucial for obtaining more local structural features.

Fig. 3(a) and (b) provide the Raman spectra of unpoled and poled samples, respectively. Fig. 3(c) shows the Lorentzian peak deconvolutions of unpoled SBTO sample as an example. Eight Raman modes can be observed in the studied wavenumber range, and these modes can be divided into three regions: (region $A-A$ ) the wavenumber below $200 \mathrm{~cm}^{-1}$ assigned as the vibration of $A$-site cations; (region $B-B_{1}, B_{2}$ ) the wavenumber range of $200-400 \mathrm{~cm}^{-1}$ related to the Ti-O vibration; (region $C-C_{1}, C_{2}, C_{3}, C_{4}, C_{5}$ ) the high frequency above $400 \mathrm{~cm}^{-1}$ related to the vibration involving oxygen displacement. ${ }^{6,7}$ It can be seen that the unpoled samples exhibit similar Raman spectra regardless of SBT content. After the poling treatment, however, clear evolutions can be distinguished for $B_{2}$ and $C_{3}$ modes as marked by the dashed lines and dark arrows in Fig. 3(b). Fig. 3(d) shows the intensity ratios of $B_{2}$ and $B_{1}$ modes $\left(I_{\mathrm{B} 2} / I_{\mathrm{B} 1}\right), C_{3}$ and $B_{1}$ modes $\left(I_{\mathrm{C} 3} / I_{\mathrm{B} 1}\right)$ as a function of SBT content. The intensity ratios present a clear difference between the unpoled and poled SBTO-SBT4 samples, indicating a poling-induced irreversible transition. ${ }^{5,33}$ However, this difference reduces progressively with increasing SBT content and nearly vanishes for SBT5 critical composition. That is to say, the poling process cannot induce any local structural changes, and the field-induced transition is essentially reversible for SBT5-SBT10 samples., ${ }^{5,33}$

To further investigate the thermal evolution of local structure, we collected the temperature dependent Raman spectra of poled SBTO, SBT2 and SBT5 samples, as shown in Fig. 4(a)-(c), respectively. The corresponding contour maps are presented in Fig. 4(d)-(f), respectively. Fig. S8 summarizes the detailed variations of wavenumber, peak-width and intensity of $B_{1}, B_{2}$ and $C_{3}$ modes with increasing temperature. Note that the as-measured Raman spectra have been corrected by the Bose-Einstein population factor: $n(w)+1=1 /\left[1-\exp \left(-\hbar w / k_{\mathrm{B}} T\right)\right]$, where $\hbar$ and $k_{\mathrm{B}}$ are Planck's and Boltzmann's constants, respectively. $^{36}$ It is known that the continuous phonon softening due to the reduced lattice polarity can be expected if no phase transition appears. ${ }^{6,7,36}$ However, the discontinuity in Raman peaks reveals the message of local transition. ${ }^{6,7,36}$ As shown in Fig. 4 and Fig. S8, an abrupt change on Raman peaks appears for poled SBTO and SBT2 samples at their $T_{\mathrm{FR}}$, especially the significant increase of Raman intensity. However, the Raman spectra of poled SBT5 sample only experience monotonic variation upon heating. This is in conformity with the lower-temperature dielectric anomalies. The poling-induced ferroelectric constrained state is broken into a more cubic state (relaxor state) by temperature, resulting in the discontinuities on dielectric and Raman spectra at $T_{\mathrm{FR}}$ for SBTO and SBT2 samples. ${ }^{7}$ However, the $T_{\mathrm{FR}}$ of SBT5 sample shifts to near or below room temperature, thus no sudden changes can be observed.

The local poling experiment was conducted to explore the possibility of inducing transformation into a local ferroelectric state. Fig. 5 shows the OP PFM amplitude and phase images of SBT0, SBT2, SBT5 and SBT10 compositions. The positive $(3 \times 3$ $\mu \mathrm{m}^{2}$ area $)$ and negative voltage $\left(1 \times 1 \mu \mathrm{m}^{2}\right.$ area $)$ of $\pm 10 \mathrm{~V}$ was simultaneously applied to check for domain reversibility, and the PFM mappings were recorded after the removal of $\mathrm{dc}$ voltage. For SBTO sample, the applied voltage is sufficient to induce the macro-domains, where the clear contrast between interior and external square area appears. This clearly indicates an irreversible field-induced ferroelectricity on the microscale, ${ }^{37,38}$ in accordance with the macroscopically measured electromechanical properties. For SBT2 sample, only partial domains can be induced, indicated by the dim and incomplete contrast. For SBT5 and SBT10 samples, domains cannot be written and the amplitude nearly disappears, revealing that the switched domains may quickly relax back to the initial state. ${ }^{37,38}$ This is also consistent with the macroscopic observation of their significantly reduced piezoelectric $d_{33}$ values. The PFM results clearly signify the enhanced domain relaxation behavior with increasing SBT content.

\subsection{In-situ polarization current response}

To further explore the composition- and temperaturedependence of polarization switching mechanisms, the in-situ polarization current curves with increasing temperature were measured. Fig. 6(a) shows the room-temperature (RT) I-E loops of SBT0, SBT2 and SBT5 samples. Two current peaks $\left(P_{1}\right)$ can be observed for SBTO sample, indicating the typical domain switching behavior of normal ferroelectrics. ${ }^{21-24,39}$ For SBT2 sample, two additional current peaks $\left(P_{2}\right)$ appear at lower-field region, suggesting the presence of relaxor phase and a twostep transition processes during loading or unloading of external field: the $P_{2}$ peak corresponds to the ferroelectricrelaxor transition with the disruption of polar domains into nanodomains, while the $P_{1}$ peak indicates the relaxorferroelectric transition with the coalescence and re-formation of large domains. ${ }^{21-24,39-41}$ Furthermore, the increase in fraction of ergodic relaxor phase moves the $P_{2}$ peak to near zero field for SBT5 sample, similar to those of SBTO and SBT2 samples at 
their $T_{\mathrm{FR}}$ (inset of Fig. 6(a)), revealing the critical transition nature of SBT5 composition. ${ }^{21-24,39}$

Fig. S9 shows the temperature dependence of $I-E$ loops for SBT0, SBT2 and SBT5 samples, and the corresponding contour maps are presented in Fig. 6(b)-(d), respectively. The top and bottom maps represent the electric loading and unloading processes, respectively. The dashed lines and circles mark the zero field, $T_{\mathrm{FR}}$ temperature and critical transition region. For SBTO sample, only two $P_{1}$ peaks exist below $60{ }^{\circ} \mathrm{C}$ and they gradually shift to the lower-field region with increasing temperature, suggesting a ferroelectric softening behavior. ${ }^{39}$ As the temperature increases above $60{ }^{\circ} \mathrm{C}$, two $P_{2}$ peaks appear, similar to that of SBT2 sample at RT, indicating the presence of relaxor phase. ${ }^{21-24,39}$ The $P_{2}$ peaks of SBTO and SBT2 samples move to zero field at their $T_{\mathrm{FR}}$, and this state is identical to that of SBT5 sample at RT. Above $T_{\mathrm{FR}}$, the $P_{2}$ peaks of all samples shift to the higher field region in second and fourth quadrant, revealing that the system has completely transformed into the ergodic relaxor state. ${ }^{21-24,39}$ However, the $P_{1}$ peaks become more diffuse above $T_{\mathrm{FR}}$. This can be attributed to the increased energy barrier between ergodic realxor phase and ferroelectric phase, making it more difficult to re-establish the long-range ferroelectric order at high temperatures. ${ }^{23,39}$ The polarization current measurements further evidence that the SBT substitution can effectively destroy the polar order and stabilize the relaxor phase at RT.

\subsection{Origin of giant electrostrain-role of local defects}

We now turn to the structural/physical origin of the giant electrostrain response. The chemical modifier $\left(\mathrm{Sr}_{0.8} \mathrm{Bi}_{0.1} \square_{0.1}\right) \mathrm{TiO}_{2.95}$ is the A-site complex relaxors with the coexistence of $V_{\mathrm{A}}$ and $V_{\mathrm{O}}$ to balance the charge neutrality. ${ }^{17}$ To elucidate the defect chemistry in A-site deficient system, the impedance spectroscopy, which is a powerful tool in investigating electrical conductivity mechanism of electroceramics, ${ }^{42-45}$ was carried out on all studied compositions. Fig. 7(a)-(d) show the frequency dependence of imaginary part $\left(Z^{\prime \prime}\right)$ of complex impedance for SBT0, SBT2, SBT5 and SBT10 samples, respectively, and the insets provide the imaginary part $\left(Z^{\prime \prime}\right)$ vs. real part $\left(Z^{\prime}\right)$ plots. All the samples present typically undistorted Debye-like semicircles, which are related to the bulk response. ${ }^{42-45}$ As the temperature increases from $480{ }^{\circ} \mathrm{C}$ to $560{ }^{\circ} \mathrm{C}$, the $Z^{\prime \prime}$ peaks move to higher frequencies and the impedance semicircles shrink successively, which is a characteristic of thermally activated relaxation process. $^{42-45}$ In addition, the activation energy $E_{\mathrm{a}}$ can be calculated by the Arrhenius format: $f_{\max }=f_{0} \exp \left(-E_{\mathrm{a}} / k T\right)$, where $f_{\max }$ is the frequency of $Z^{\prime \prime}$ peak, $f_{0}$ is a constant, $k$ is Boltzmann constant. ${ }^{45}$ The plots of $\ln \left(f_{\max }\right)$ vs. $1000 / T$ and the fitting lines are displayed in Fig. 7(e) and (f), and the $E_{\mathrm{a}}$ values are summarized in Fig. 8(b). The $E_{\mathrm{a}}$ values of all compositions lie in the range of $0.96-1.63 \mathrm{eV}$, which are close to the activation energy of oxygen vacancies $(\sim 1 \mathrm{eV}){ }^{42-46}$ This result reveals that the present system exhibits intrinsic electronic conduction and the oxygen vacancies dominate the high-temperature conduction mechanism. ${ }^{42-46}$ The reduction of $E_{\mathrm{a}}$ values with increasing SBT content suggests the increased oxygen vacancy concentration. ${ }^{42-46}$ This is reasonable since more $V_{\mathrm{A}}$ and $V_{\mathrm{O}}$ local defects will be introduced into the material system with SBT substitution. Note that the defect content is not enough to transform the present system to an oxide-ion conductor, and it remains an insulator. ${ }^{42-46}$

The local defects are randomly distributed in crystal lattice, inducing a local polarization field to affect the thermodynamic stability of ferroelectric state. ${ }^{47}$ Based on the Landau-Devonshire theory, the free energy $(F)$ of the states without and with defects can be described by Landau polynomials:

$F=\alpha P^{2}+B P^{4}+\gamma P^{6}$, the state without defects

$F=\alpha P^{2}+B P^{4}+\gamma P^{6}-E_{\mathrm{d}} P$, the state with defects

where $\alpha, \beta$ and $\gamma$ are expansion factors, $E_{\mathrm{d}}$ is the local defect field caused by $V_{\mathrm{A}}$ and $V_{\mathrm{O}}, P$ is the order parameter, i.e., the spontaneous polarization. ${ }^{48-50}$ Fig. 8 (c) and (d) present the schematic Landau free energy with respect to the polarization for the states without and with defects, respectively. It is clearly that the defects couple strongly to the polarization and break the symmetry of Landau potential curves. Fig. 8(a) shows the structural model of the giant electrostrain. The SBTO sample behaves like normal ferroelectrics with larger-size frozen domains, and the external field can irreversibly transform the system into the ferroelectric state, characterized by a large piezoelectric $d_{33}$ value, high remanent polarization and low strain aforementioned. ${ }^{5,15}$ The introduced local defects prefer certain polarizations at randomly distributed defect locations, which impede the development of long-range polar correlation and favor the presence of relaxor phase with dynamic and weakly correlated PNRs. ${ }^{18,47,48}$ For SBT5 critical composition, the $T_{\mathrm{FR}}$ moves to around room temperature, probably resulting in the coexistence of ferroelectric and relaxor order. ${ }^{51}$ When applying a sufficiently high external field, the energy fluctuation of the system is suppressed and the long-range ferroelectric ordered state can be reconstructed in non-polar matrix by merging discrete PNRs. ${ }^{48,52}$ After the applied field is released, the system will quickly relax back to initial state with the aid of local defect field, producing a giant recoverable strain in SBT5 composition. ${ }^{48,52}$ With the defect concentration exceeds a critical value, the long-range dipole alignment is difficult to obtain from relaxor matrix since the strong defect fields inhibit the aggregation of PNRs, ${ }^{18,47,48}$ which is responsible for the deterioration of strain performance of SBT6-SBT10 samples.

The low driving field amplitude of the giant electrostrain for SBT5 sample can be explained as follows. Based on the electromechanical and structural characterizations aforementioned, the critical composition SBT5 locates at the ferroelectric-relaxor transitional boundary, and a slight residual ferroelectric (or quasi-ferroelectric) phase still preserves. ${ }^{51}$ This weak ferroelectric order may act as seed for 
the growth of polar domains and help the system skip the nucleation process. ${ }^{48,51}$ Furthermore, the introduced local defects possess the lowest potential barrier and the free energy of material system can be effectively lowered. ${ }^{47-50,53}$ The existence of these local defects can facilitate the growth of ferroelectric domains under a given external field. ${ }^{47-50,53}$ These synergistic contributions help reduce the electric-field required to trigger the formation of long-range polar phase and also the giant electrostrain in SBT5 composition.

\section{Conclusions}

In this work, a giant $d_{33}{ }^{*}$ value of $810 \mathrm{pm} / \mathrm{V}$ at a low driving field of $4 \mathrm{kV} / \mathrm{mm}$ was achieved in SBT5 sample. The SBT modification induces a local polarization field, which couples strongly to the polarization and favors the presence of relaxor phase. The $T_{\mathrm{FR}}$ of SBT5 sample shifts to near room temperature, engendering a large recoverable strain. The remanent ferroelectric phase and local defects are constructive for lowering the driving field of giant electrostrain in SBT5 sample. For uncovering the underlying mechanisms, the structural transitions were investigated by various techniques. The electromechanical measurements indicate a composition-induced ferroelectric-relaxor transition with SBT substitution. The Raman scattering and PFM mapping clearly signify the enhanced local structural disorder and domain relaxation behavior with increasing SBT content. The in-situ polarization current responses further evidence that the SBT can effectively stabilize the high-temperature relaxor phase at room temperature. This finding provides a promising approach to achieve a low-field-driven large strain by introducing local defects. Certainly, the present strategy may also be helpful for tailoring performances of other lead-free piezoelectrics by means of microstructure engineering.

\section{Conflicts of interest}

There are no conflicts to declare.

\section{Acknowledgements}

The authors would like to acknowledge the National Natural Science Foundation of China under grant No. 51772211, 51332003 and 51372171 . This work was also supported by Shanghai Municipal Science and Technology Commission funded international cooperation project under No. 16520721500 .

\section{References}

1 N. Setter and R. Waser, Acta Mater., 2000, 48, 151.

2 G. H. Haertling, J. Am. Ceram. Soc., 1999, 82, 797.

3 J. Wu, D. Xiao and J. Zhu, Chem. Rev., 2015, 115, 2559.

4 W. Liu and X. Ren, Phys. Rev. Lett., 2009, 103, 257602.
5 W. Jo, R. Dittmer, M. Acosta, J. Zang, C. Groh, E. Sapper, K. Wang and J. Rödel, J. Electroceram., 2012, 29, 71.

6 B. W. Eerd, D. Damjanovic, N. Klein, N. Setter and J. Trodahl, Phys. Rev. B, 2010, 82, 104112.

7 D. Schütz, M. Deluca, W. Krauss, A. Feteira, T. Jackson and K. Reichmann, Adv. Funct. Mater., 2012, 22, 2285.

8 B. J. Chu, D. R. Chen, G. R. Li and Q. R. Yin, J. Eur. Ceram. Soc., 2002, 22, 2115.

9 W. C. Lee, Y. F. Lee, M. H. Tseng, C. Y. Huang and Y. C. Wu, J. Am. Ceram. Soc., 2009, 92, 1069.

10 A. B. Kounga, S. T. Zhang, W. Jo, T. Granzow and J. Rödel, Appl. Phys. Lett., 2008, 92, 222902.

11 S. Kim, H. Choi, S. Han, J. S. Park, M. H. Lee, T. K. Song, M. H. Kim, D. Do and W. J. Kim, J. Eur. Ceram. Soc., 2017, 37, 1379.

12 D. Maurya, M. Murayama, A. Pramanick, W. T. Reynolds, K. An and S. Priya, J. Appl. Phys., 2013, 113, 114101.

13 S. T. Zhang, A. B. Kounga, E. Aulbach, H. Ehrenberg and J. Rödel, Appl. Phys. Lett., 2007, 91, 112906.

14 J. Rödel, K. G. Webber, R. Dittmer, W. Jo, M. Kimura and D. Damjanovic, J. Eur. Ceram. Soc., 2015, 35, 1659.

15 W. Jo, T. Granzow, E. Aulbach, J. Rödel and D. Damjanovic, J. Appl. Phys., 2009, 105, 094102.

16 H. Simons, J. E. Daniels, J. Glaum. A. J. Studer, J. L. Jones and M. Hoffman, Appl. Phys. Lett., 2013, 102, 062902.

17 C. Ang and Z. Yu, J. Appl. Phys., 2002, 91, 1487.

18 D. Wang, X. Ke, Y. Wang, J. Gao, Y. Wang, L. Zhang, S. Yang and X. Ren, Phys. Rev. B, 2012, 86, 054120.

19 R. D. Shannon, Acta Cryst., 1976, A32, 751.

20 R. A. Malik, J. K. Kang, A. Hussain, C. W. Ahn, H. S. Han and J. S. Lee, Appl. Phys. Express., 2014, 7, 061502.

21 G. Viola, R. Mckinnon, V. Koval, A. Adomkevicius, S. Dunn and H. Yan, J. Phys. Chem. C, 2014, 118, 8564.

22 H. S. Han, W. Jo, J. K. Kang, C. W. Ahn, I. W. Kim, K. K. Ahn and J. S. Lee, J. Appl. Phys., 2013, 113, 154102.

23 X. Liu, F. Li, P. Li, J. Zhai, B. Shen and B. Liu, J. Eur. Ceram. Soc., 2017, 37, 4585 .

24 A. Maqbool, A. Hussain, R. A. Malik, J. U. Rahman, A. Zaman, T. K. Song, W. J. Kim and M. H. Kim, Mater. Sci. Eng. B, 2015, 199, 105.

25 W. Zhao, R. Zuo, J. Fu and M. Shi, J. Eur. Ceram. Soc., 2014, 34, 2299.

26 W. Zhao, R. Zuo and J. Fu, J. Eur. Ceram. Soc., 2104, 34, 4235.

27 D. Zheng and R. Zuo, J. Eur. Ceram. Soc., 2015, 35, 3485.

28 K. Wang, F. Z. Yao, W. Jo, D. Gobeljic, V. V. Shvartsman, D. C. Lupascu, J. F. Li and J. Rödel, Adv. Funct. Mater., 2013, 23, 4079.

29 Y. Saito, H. Takao, T. Tani, T. Nonoyama, K. Takatori, T. Homma, T. Nagaya and M. Nakamura, Nature, 2004, 432, 84

30 M. H. Zhang, K. Wang, J. S. Zhou, J. J. Zhou, X. Chu, X. Lv, J. Wu and J. F. Li, Acta Mater., 2017, 122, 344.

31 M. H. Zhang, K. Wang, Y. J. Du, G. Dai, W. Sun, G. Li, D. Hu, H. C. Thong, C. Zhao, X. Q. Xi, Z. X. Yue and J. F. Li, J. Am. Chem. Soc., 2017, 139, 3889.

32 H. Qi, R. Zuo, D. Zheng and A. Xie, J. Alloy. Compd., 2017, 724, 774.

33 W. Jo, S. Schaab, E. Sapper, L. A. Schmitt, H. J. Kleebe, A. J. Bell and J. Rödel, J. Appl. Phys., 2011, 110, 074106.

34 D. I. Woodward, R. Dittmer, W. Jo, D. Walker, D. S. Keeble, M. W. Dale, J. Rödel and P. A. Thomas, J. Appl. Phys., 2014, 115, 114109.

35 K. Datta, P. A. Thomas and K. Roleder, Phys. Rev. B, 2010, 82 224105.

36 N. Waeselmann, B. Mihailova, B. J. Maier, C. Paulmann, M. Gospodinov, V. Marinova and U. Bismayer, Phys. Rev. B, 2011, 83, 214104.

37 R. Dittmer, W. Jo, J. Rödel, S. Kalinin and N. Balke, Adv. Funct. Mater., 2012, 22, 4208. 
38 Q. Yao, F. Wang, F. Xu, C. M. Leung, T. Wang, Y. Tang, X. Ye, Y. Xie, D. Sun and W. Shi, ACS Appl. Mater. Interfaces., 2015, 7, 5066.

39 Y. Ehara, N. Novak, S. Yasui, M. Itoh and K. G. Webber, Appl. Phys. Lett., 2015, 107, 262903.

40 W. Jo, J. Daniels, D. Damjanovic, W. Kleemann and J. Rödel, Appl. Phys. Lett., 2013, 102, 192903.

41 H. Guo, X. Liu, F. Xue, L. Q. Chen, W. Hong and X. Tan, Phys. Rev. B, 2016, 93, 174114.

42 M. Li, H. Zhang, S. N. Cook, L. Li, J. A. Kilner, I. M. Reaney and D. C. Sinclair, Chem. Mater., 2015, 27, 629.

43 L. Li, M. Li, H. Zhang, I. M. Reaney and D. C. Sinclair, J. Mater. Chem. C, 2016, 4, 5779.

44 F. Yang, P. Wu and D. C. Sinclair, J. Mater. Chem. C, 2017, 5, 7243.

45 Z. Liu, H. Fan, Y. Zhao and G. Dong, J. Am. Ceram. Soc., 2016, 99, 146.

46 B. S. Kang, S. K. Choi and C. H. Park, J. Appl. Phys., 2003, 94, 1904.

47 S. Jesse, B. J. Rodriguez, S. Choudhury, A. P. Baddorf, I. Vrejoiu, D. Hesse, M. Alexe, E. A. Eliseev, A. N. Morozovska, J. Zhang, L. Q. Chen and S. V. Kalinin, Nat. Mater., 2008, 7, 209.

48 T. Li, X. Lou, X. Ke, S. Cheng, S. Mi, X. Wang, J. Shi, X. Liu, G. Dong, H. Fan, Y. Wang, X. Tan, Acta Mater., 2017, 128, 337.

49 Y. Zhou, D. Xue, X. Ding, L. Zhang, J. Sun and X. Ren, J. Phys.: Condens. Matter., 2013, 25, 435901.

50 D. Wang, Y. Wang, Z. Zhang and X. Ren, Phys. Rev. Lett., 2010, 105, 205702.

51 H. S. Han, W. Jo, J. Rödel, I. K. Hong, W. P. Tai and J. S. Lee, J. Phys.: Condens. Matter., 2012, 24, 365901.

52 J. Fu and R. Zuo, Acta Mater., 2013, 61, 3687.

53 D. Viehland and J. F. Li, J. Appl. Phys., 2001, 90, 2995. 


\section{Figure captions}

Fig. 1 The $P$-E hysteresis loops (a), bipolar (b) and unipolar (c) S-E curves of SBTO-SBT10 ceramics. Unipolar strain curves of SBT5 ceramics measured at different electric field (d). Comparison of several actuator ceramics against their $d_{33}{ }^{*}$ values and driving fields ${ }^{14,25-31}(e)$. The $d S / d E$ values of the lower-half $S$ - $E$ curves during electric loading (f).

Fig. 2 The temperature dependence of dielectric constant $\left(\varepsilon_{\mathrm{r}}\right)$ and loss tangent $(\tan \delta)$ of poled SBTO-SBT5 samples upon heating (a). The depolarization current $j_{\mathrm{TSDC}}$ as a function of temperature for poled SBTO-SBT5 samples (b). Composition-temperature phase diagram of the present system (c). The diffuseness degree $n$ and $\Delta T$ values with varying the SBT content (d).

Fig. 3 Raman spectra of unpoled (a) and poled (b) SBTO-SBT10 samples. The Lorentzian deconvolutions of unpoled SBT0 sample (c). The intensity ratios of $B_{2}$ and $B_{1}$ modes $\left(I_{\mathrm{B} 2} / I_{\mathrm{B} 1}\right), C_{3}$ and $B_{1}$ modes $\left(I_{\mathrm{C} 3} / I_{\mathrm{B} 1}\right)$ as a function of SBT content $(\mathrm{d})$.

Fig. 4 The in-situ temperature dependence of Raman spectra (a)-(c) and the corresponding contour maps (d)-(f) for poled SBT0, SBT2 and SBT5 samples, respectively.

Fig. 5 The out-of-plane PFM amplitude and phase images $\left(5 \times 5 \mu \mathrm{m}^{2}\right)$ of SBTO, SBT2, SBT5 and SBT10 samples under the voltage of $\pm 10 \mathrm{~V}$ in $3 \times 3$ and $1 \times 1 \mu \mathrm{m}^{2}$ areas.

Fig. 6 The room-temperature I-E loops of SBTO, SBT2 and SBT5 samples (a), inset shows the I-E loops of SBT0 and SBT2 samples at their $T_{\mathrm{FR}}$. The contour maps of $I-E$ loops as a function of temperature for SBTO (b), SBT2 (c) and SBT5 (d) samples, respectively. The top and bottom maps correspond to the electric loading and unloading processes, respectively.

Fig. 7 The impedance spectra of SBTO (a), SBT2 (b), SBT5 (c) and SBT10 (d) samples. Data was collected in the frequency range of $150 \mathrm{~Hz}-2 \mathrm{MHz}$ and the temperature range of $480{ }^{\circ} \mathrm{C}-560{ }^{\circ} \mathrm{C}$. $\ln \left(f_{\max }\right)$ vs. $1000 / T$ plots of SBTO-SBT10 samples (symbol: experimental data, solid lines: fitting lines) (e)(f).

Fig. 8 Model of the giant strain response in A-site deficient NBT-BZT-xSBT system (a). The calculated activation energy $E_{\mathrm{a}}$ values as a function of SBT content (b). Schematic Landau potential curves of the states without (c) and with (d) defects, respectively. 

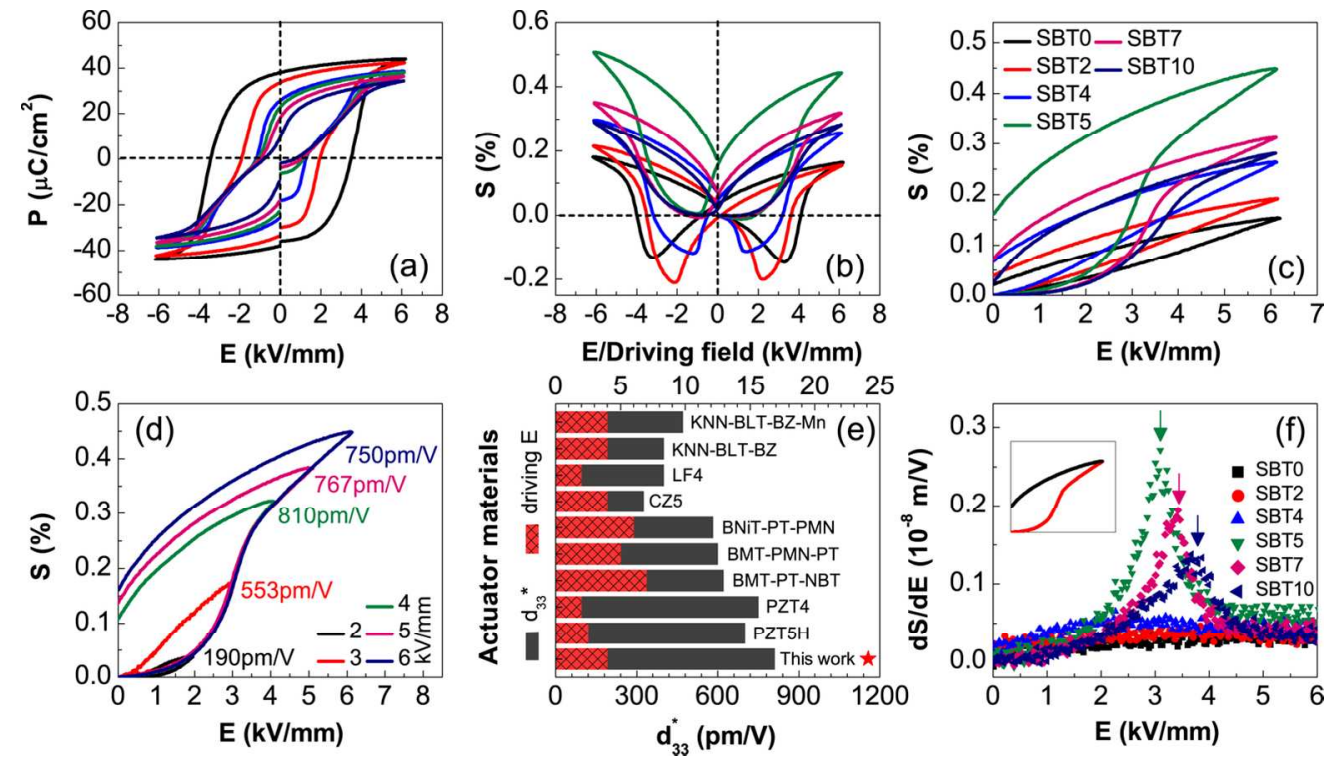

Fig. 1 The P-E hysteresis loops (a), bipolar (b) and unipolar (c) S-E curves of SBT0-SBT10 ceramics. Unipolar strain curves of SBT5 ceramics measured at different electric field (d). Comparison of several actuator ceramics against their d33* values and driving fields14,25-31 (e). The dS/dE values of the lowerhalf S-E curves during electric loading (f). 



Fig. 2 The temperature dependence of dielectric constant ( $\varepsilon r$ ) and loss tangent (tan $\delta$ ) of poled SBT0-SBT5 samples upon heating (a). The depolarization current jTSDC as a function of temperature for poled SBTOSBT5 samples (b). Composition-temperature phase diagram of the present system (c). The diffuseness degree $\mathrm{n}$ and $\Delta \mathrm{T}$ values with varying the SBT content (d). 

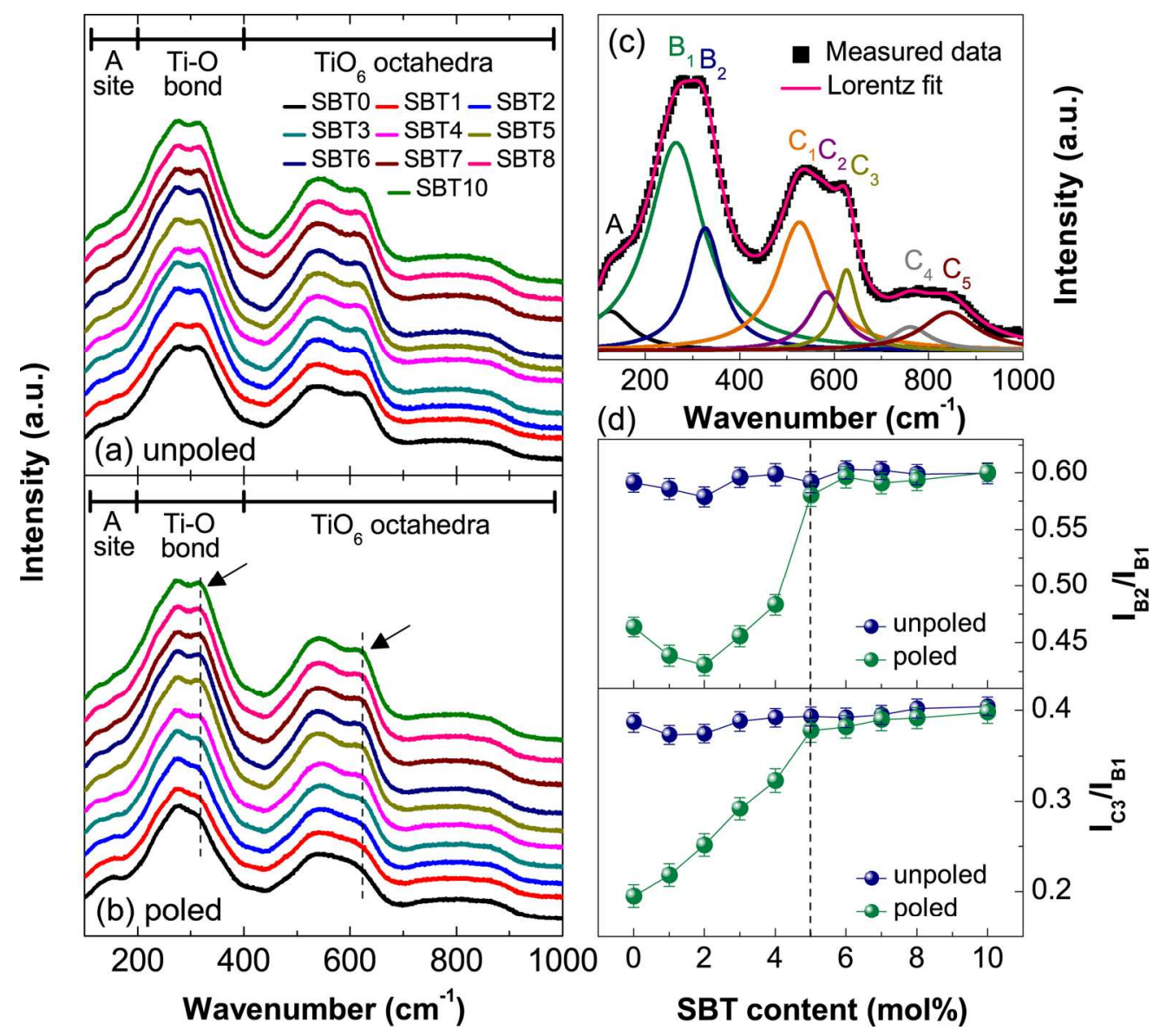

Fig. 3 Raman spectra of unpoled (a) and poled (b) SBT0-SBT10 samples. The Lorentzian deconvolutions of unpoled SBT0 sample (c). The intensity ratios of B2 and B1 modes (IB2/IB1), C3 and B1 modes (IC3/IB1) as a function of SBT content (d). 

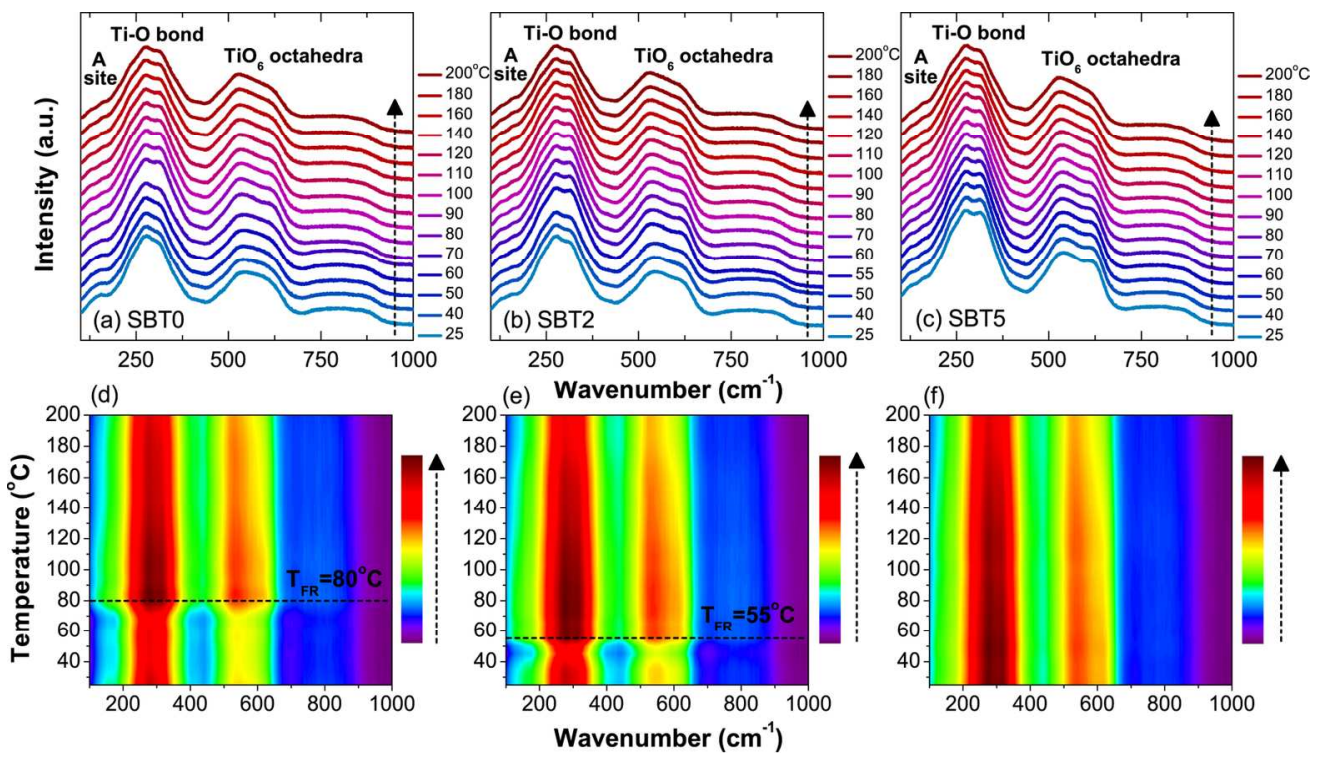

Fig. 4 The in-situ temperature dependence of Raman spectra (a)-(c) and the corresponding contour maps (d)-(f) for poled SBT0, SBT2 and SBT5 samples, respectively.

$150 \times 85 \mathrm{~mm}(300 \times 300 \mathrm{DPI})$ 


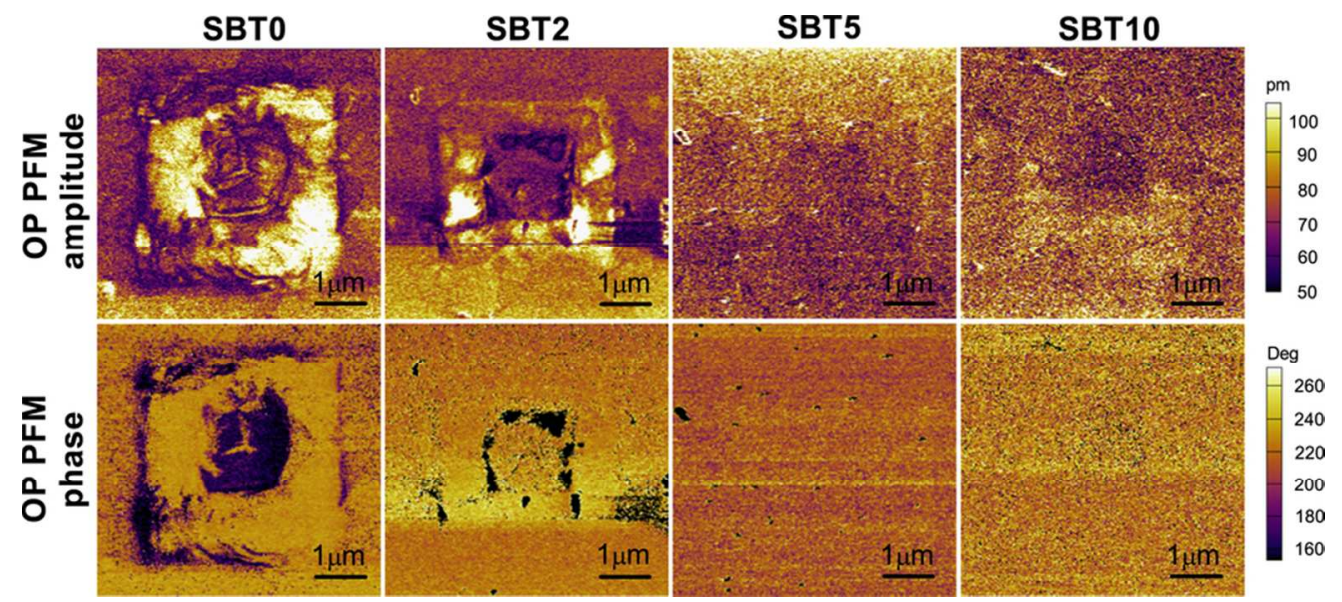

Fig. 5 The out-of-plane PFM amplitude and phase images $(5 \times 5 \mu \mathrm{m} 2)$ of SBT0, SBT2, SBT5 and SBT10 samples under the voltage of $\pm 10 \mathrm{~V}$ in $3 \times 3$ and $1 \times 1 \mu \mathrm{m} 2$ areas.

$83 \times 37 \mathrm{~mm}(300 \times 300 \mathrm{DPI})$ 

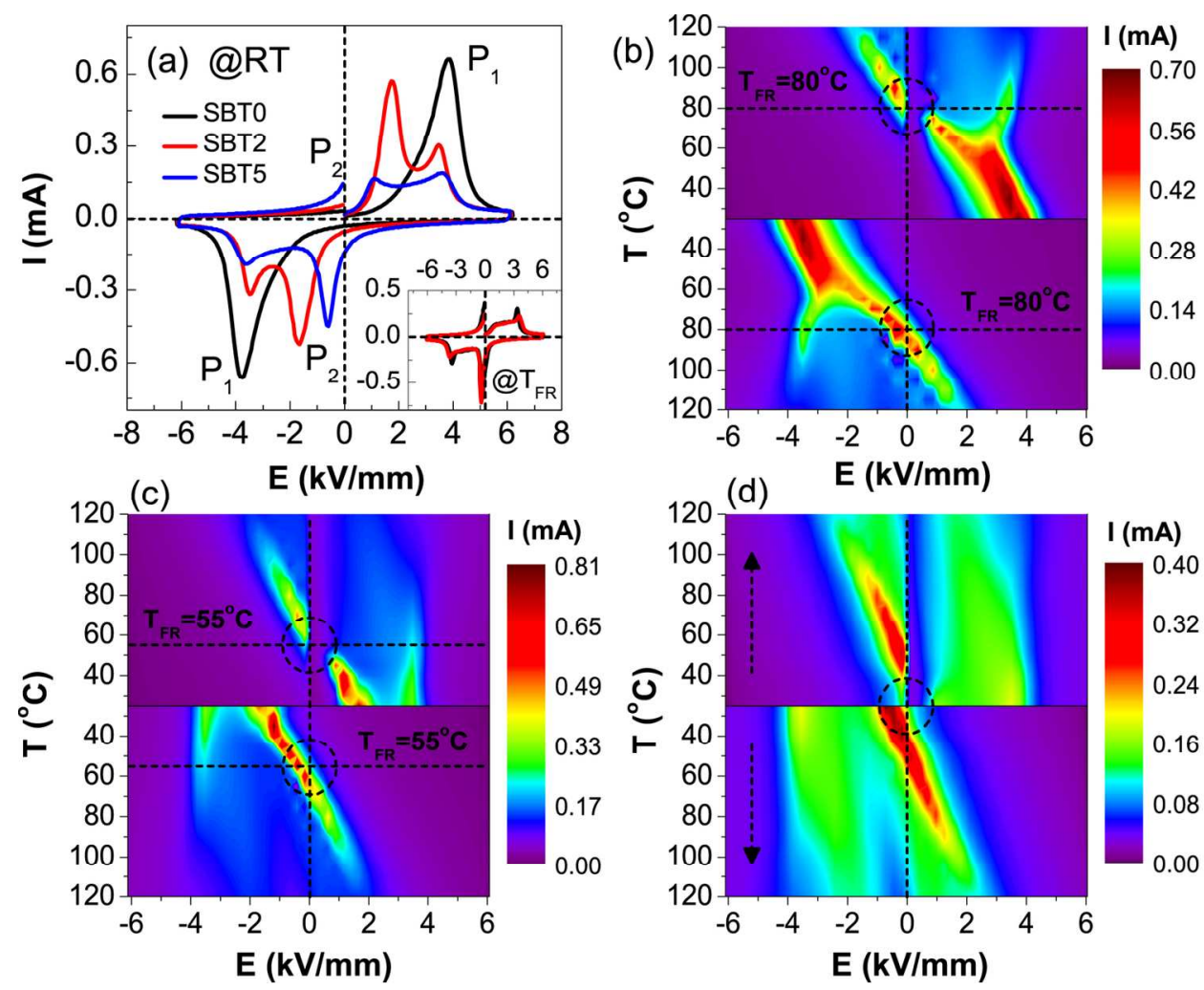

Fig. 6 The room-temperature I-E loops of SBT0, SBT2 and SBT5 samples (a), inset shows the I-E loops of SBT0 and SBT2 samples at their TFR. The contour maps of I-E loops as a function of temperature for SBT0 (b), SBT2 (c) and SBT5 (d) samples, respectively. The top and bottom maps correspond to the electric loading and unloading processes, respectively. 

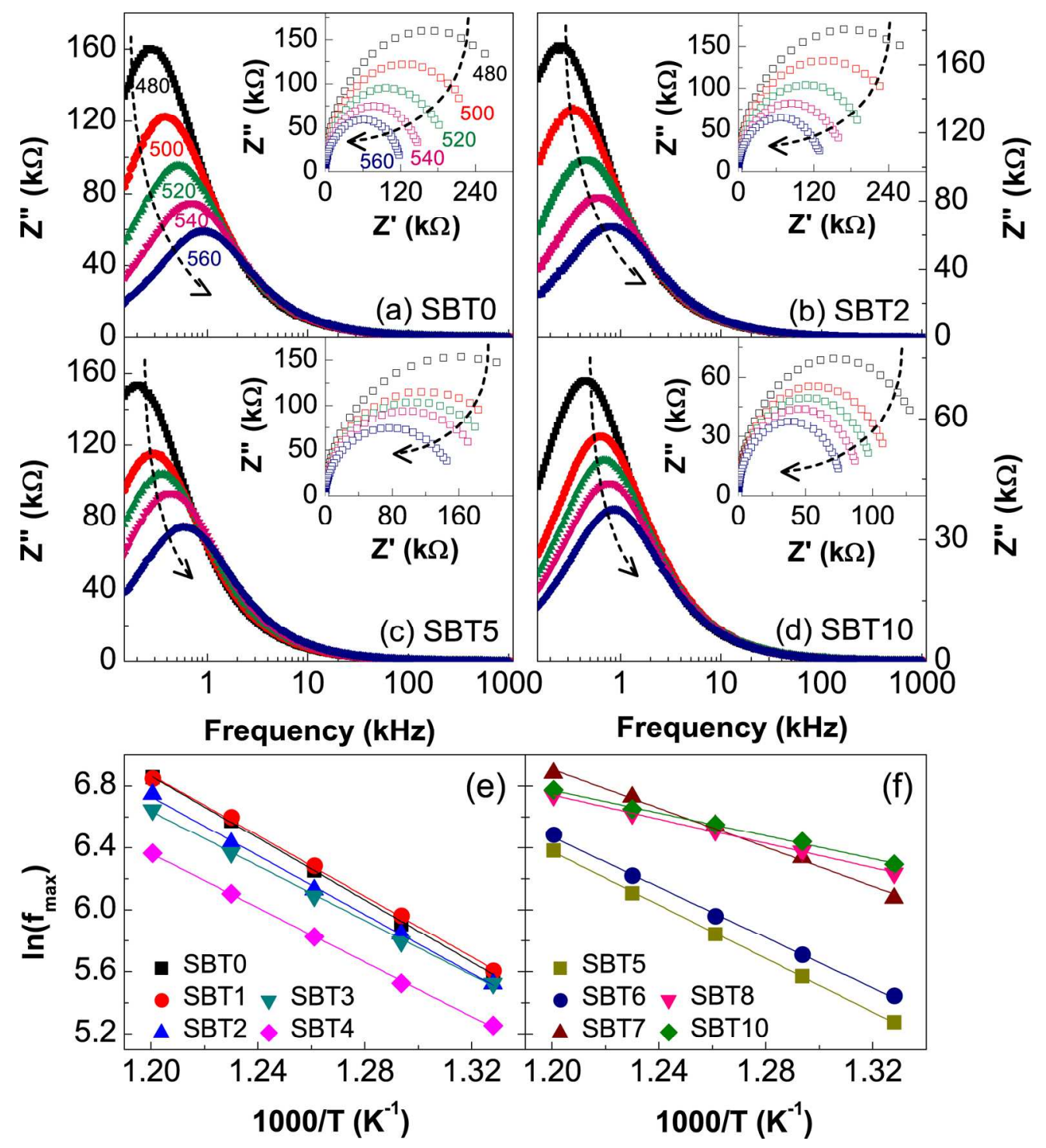

Fig. 7 The impedance spectra of SBT0 (a), SBT2 (b), SBT5 (c) and SBT10 (d) samples. Data was collected in the frequency range of $150 \mathrm{~Hz}-2 \mathrm{MHz}$ and the temperature range of $480^{\circ} \mathrm{C}-560^{\circ} \mathrm{C}$. In(fmax) vs. $1000 / \mathrm{T}$ plots of SBT0-SBT10 samples (symbol: experimental data, solid lines: fitting lines) (e)(f). 
(a) SBT0
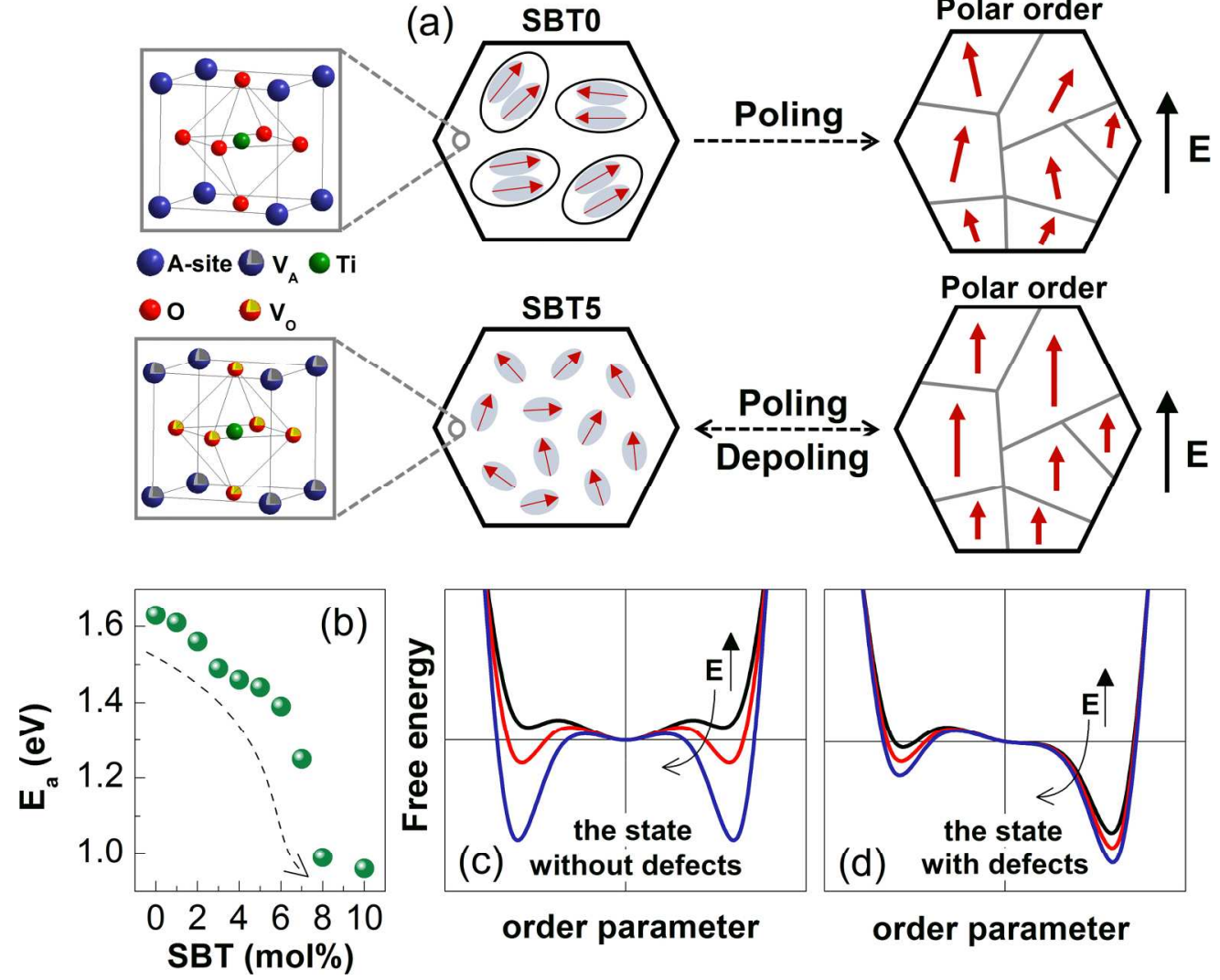

order parameter

Fig. 8 Model of the giant strain response in A-site deficient NBT-BZT-xSBT system (a). The calculated activation energy Ea values as a function of SBT content (b). Schematic Landau potential curves of the states without (c) and with (d) defects, respectively. 


\section{Table of Contents Entry}

Lead-free NBT-based piezoceramics: local defects boost ferroelectric instability and giant electrostrain

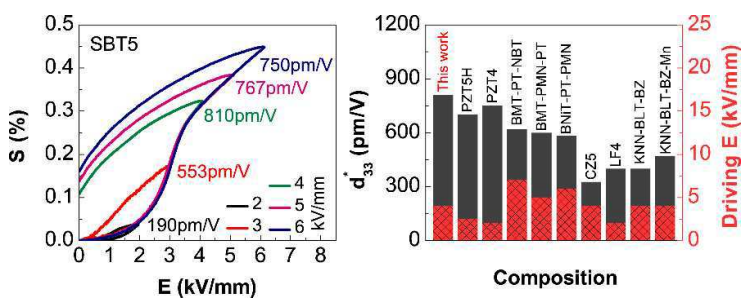

